\title{
CAPITALISMO GLOBAL Y ESTADO NACIONAL en las luchas de los cultivos transgénicos en Brasil
}

\section{Renata Motta}

Resumen: La introducción de la biotecnología es parte de un proceso global de cambio estructural en la agricultura caracterizado por una cada vez mayor integración de la agricultura mundial con alto control corporativo. Sin embargo, ya que la competencia legal para permitir el cultivo y el comercio de cultivos genéticamente modificados (GM) comúnmente se encuentra a nivel del Estado nacional, éste continúa siendo estratégico en la política de transgénicos tanto para actores promotores de la tecnología como para movimientos sociales que pelean contra ella. El presente artículo ilustra este argumento con un análisis de las luchas acerca de los cultivos transgénicos en Brasil. Se demuestra cómo la implementación de un régimen alimentario basado en biotecnología, control corporativo y globalismo neoliberal dependió del Estado y fue un proceso disputado.

Palabras clave: Brasil, Estado, régimen alimentario, movimientos sociales, transgénicos.

\footnotetext{
"Universidad Libre de Berlín, Instituto Latinoamericano, Rüdesheimerstr, Alemania. Correo-e: renata.motta@fu-berlin.de
} 


\section{GLOBAL CAPITALISM AND THE NATION STATE in the struggles over GM crops in Brazil}

Abstract: The introduction of biotechnology is part of a global process of structural change in agriculture characterized by an increased integration of world agriculture with high corporate control. However, as the legal competence to allow the planting and trade of genetically modified (GM) crops commonly lies at the level of the nation state, this remains strategic in the politics of GM crops, both for actors promoting the technology and for social movements struggling against it. This paper illustrates this argument with an analysis of the struggles over GM crops in Brazil. It shows how the implementation of a food regime based on biotechnology, corporate control and neoliberal globalism depended on the state and was a contested process.

Keywords: Brazil, state, food regime, social movements, transgenic. 


\section{Introducción}

La biotecnología es un elemento constitutivo de la base agraria de la economía mundial (Kloppenburg, 2004). Las semillas genéticamente modificadas (GM) han sido instrumentales para la reproducción de la dependencia económica y de las asimetrías de poderes entre países y para garantizar la participación dominante de las corporaciones transnacionales. Debido a su mayor biodiversidad y por ser el centro de origen de la mayoría de los cultivos básicos consumidos mundialmente, los países menos desarrollados del Sur global transfieren recursos genéticos al Norte industrializado, mientras pocas corporaciones concentran las patentes de la mayoría de las semillas GM disponibles (Fuglie et al., 2012). Dado ese nivel de control, McMichael (2009) caracteriza la formación agraria actual como «régimen alimentario corporativo». En realidad las corporaciones biotecnológicas y sus redes transnacionales son las promotoras más activas de los cultivos transgénicos (Glover, 2010; Gras y Hernández, 2008) y se transformaron fácilmente en objeto del activismo transnacional (Schurman y Munro, 2010).

A fin de lograr una integración completa de la agricultura con fundamento en las dinámicas del mercado global es necesaria una serie de regulaciones. Lo anterior no es una característica nueva del capitalismo agrario; desde hace tiempo analistas de los regímenes alimentarios (Friedmann \& McMichael, 1989) han argumentado que la base material de los mercados agrarios depende de un sistema de Estados y de sus bases institucionales. En el caso de la biotecnología, la negociación de instrumentos legales internacionales ha sido fundamental en la creación de las condiciones apropiadas para su adopción y difusión (Góngora y Motta, 2014; Pechlaner \& Otero, 2008). Éstas incluyen regulaciones de libre comercio que fortalecen los derechos de propiedad intelectual (DPI) y una cultura política internacional basada en la ciencia y el riesgo como 
criterios en la toma de decisiones (Motta, 2015). Si bien ha permanecido bajo la influencia de corporaciones, las regulaciones de libre comercio han sido crecientemente impugnadas por movimientos agrarios transnacionales (Borras et al., 2008).

Sin embargo, dichas regulaciones son al final el resultado de decisiones tomadas por los Estados, en su calidad de miembros de organizaciones intergubernamentales. En ese sentido, además de las estructuras globales que promueven la biotecnología, de las corporaciones y los activistas transnacionales que disputan el futuro de los cultivos transgénicos, el nivel nacional y el Estado desempeñan también una función central en los procesos mencionados. El presente artículo destaca las disputas políticas manifiestas en el ámbito nacional, asimismo analiza cómo actores protransgénicos y movimientos sociales señalan que el Estado nacional influye el curso de la agrobiotecnología en Brasil. ${ }^{1} \mathrm{El}$ artículo está estructurado en dos partes, la presentación del argumento, seguida por la ilustración del caso brasileño.

\section{El Estado nacional}

Al evaluar el estado actual de investigaciones en torno al cambio agrario, Borras (2009: 20) identifica entre las corrientes principales tendencias que despolitizan la investigación y las prácticas políticas, ya sea por adoptar conceptos administrativos tales como «gobernanza» o bien por igualar la política a fenómenos negativos como corrupción y Estados fallidos. Tales

${ }^{1}$ Este texto es parte de una investigación más amplia (Motta, 2016) y se basa en diferentes tipos de datos recopilados para el periodo de 1996-2013. Incluye bancos de datos oficiales, fuentes documentales, literatura secundaria, artículos de diarios, documentos de campaña de movimientos sociales y datos primarios recopilados en entrevistas semiestructuradas (las cuales incorporaron activistas de los movimientos campesino-ambiental, agroecológico); derechos del consumidor, humanos y científicos. 
estudios contribuyen a legitimar el fin del desarrollo protagonizado por el Estado en favor de soluciones basadas en el mercado. Borras (2009) aborda la necesidad de llevar una política seria y a la vez analizar las relaciones entre Estado y sociedad en las dinámicas del cambio agrario. Las disputas sobre la introducción de la agrobiotecnología ofrecen una óptima entrada para indagar en las relaciones al considerar también la política.

Con base en lo anterior, destacan dos razones. Por un lado, el Estado nacional es «una esfera crítica para la imposición de los intereses capitalistas dominantes» (Otero, 2004: 325). Pechlaner y Otero (2008) prefieren denominar el régimen alimentario actual como «régimen alimentario neoliberal» con la intención de enfatizar la función de los Estados en la adopción e implementación de los órdenes institucionales que sirven más bien a los intereses de las corporaciones transnacionales y las elites agrarias. A excepción quizá de la Unión Europea, el Estado nación debe aprobar las semillas transgénicas y los agrotóxicos usados en su asociación, de igual modo tiene la responsabilidad de las políticas agrícola y macroeconómica que influencian las decisiones de los actores agrarios. Las políticas individuales de los diferentes países proporcionan cierto margen de maniobra para la implementación de acuerdos internacionales, según el papel conferido a la biotecnología en las estrategias de desarrollo - aunque esa autonomía sea distribuida asimétricamente entre ellos (Newell, 2006). En concreto, cada país decide acerca de la adopción de los cultivos transgénicos. Pese a los padrones globales en el régimen alimentario neoliberal vigente, existe una marcada variación, lo que dificulta la continuidad en las investigaciones a nivel del Estado nacional.

Por otro lado, el Estado también es el locus donde grupos subordinados son capaces de modificar las trayectorias del cambio agrario, no sólo porque el Estado ha sido instrumental en la implementación del régimen alimentario neoliberal, sino porque continúa siendo la esfera primordial en la que se han vigorizado los derechos que protegen dichos grupos. En 
ese sentido, únicamente es posible contrarrestar los imperativos sistémicos del Estado y del capital que tratan los transgénicos como una cuestión de innovación agrícola y cálculo de riesgos, mediante la definición de la biotecnología como un problema político, y por tanto, como un tema de justicia y derechos (Anderson, 2004). Así, el Estado continúa siendo estratégico en la creación de canales para la manifestación de derechos y demandas frente a las amenazas asociadas a los cultivos transgénicos.

Las investigaciones en torno a las controversias que generan los cultivos transgénicos impactan en la imbricación de los mercados, la ciencia y la política en contextos específicos, con diferentes trayectorias y órdenes morales (Jasanoff, 2005). Además, los estudios de los movimientos sociales coinciden con el hallazgo de que el Estado sigue siendo el principal blanco de las demandas de protestas (Tarrow, 2011). Por consiguiente, el debate público y la movilización social han desafiado la legitimidad de las dinámicas globales de mercado y del discurso científico como determinantes de las políticas nacionales para los transgénicos, con resultados múltiples (Borras et al., 2008; Kinchy, 2012; Motta, 2015; Schurman \& Munro, 2010).

Una objeción a este enfoque son los fuertes efectos sociales y ambientales de la expansión global de la biotecnología en las regiones productoras (Cáceres, 2014; Pengue, 2005), en particular respecto a las estrategias de sustento de las poblaciones en el medio rural y la manera en que lidian con tales efectos (Fitting, 2011; Lapegna, 2014). Referente a la movilización política, los Estados federados abren arenas subnacionales para las disputas (Tarrow, 2011). Sin embargo, el Estado central a escala nacional tiene jurisdicción sobre la aprobación o el rechazo de cultivos transgénicos, además las cortes federales pueden anular decisiones tomadas en otros niveles.

En síntesis: el Estado nacional sigue teniendo una participación central tanto en la implementación de un régimen alimentario basado en la 
biotecnología, el control corporativo y el globalismo neoliberal, como en las luchas por alternativas. A fin de ilustrar dicho argumento, tomaré como base un estudio empírico de las pugnas políticas en cuanto a los cultivos transgénicos en Brasil. Sin desconsiderar la integración de este tipo de disputas políticas en el sistema-mundo, analizo cómo los actores que se resisten a la adopción de cultivos transgénicos en Brasil intentan influir en la política nacional. El presente artículo evidencia la forma en que el globalismo neoliberal y los intereses de las empresas transnacionales son mediados por la política nacional, hecho que abre distintas posibilidades para el funcionamiento del régimen mundial de alimentos en determinado caso.

\section{Disputas por los cultivos transgénicos en Brasil}

Según datos de la industria biotecnológica (James, 2015), Brasil es actualmente el segundo productor más grande de transgénicos, con 42.2 millones de hectáreas plantadas en 2014. ${ }^{2}$ No obstante, lograr esa posición no ha sido tan fácil. Fue apenas en 2009 que la mayoría de los campos de soja brasileños fueron convertidos a cultivos transgénicos, un proceso que ya había tenido lugar en Argentina diez años antes. La diferencia se explica, en gran medida, por la movilización social contra ellos. Aunque se pensó que Brasil seguiría el padrón establecido por Estados Unidos y Argentina, hubo desconcierto al encontrar una fuerte oposición de organizaciones de la sociedad civil, movimientos sociales y gobiernos subnacionales, de ahí la pertinencia de analizar este país. Las disputas serán narradas en cinco pasos: globalismo neoliberal, cultivos ilegales, movilización social, lobby rural y comisión de expertos.

${ }^{2}$ Estados Unidos lidera la clasificación con 73.1 millones de hectáreas, le sigue Argentina con 24.3 millones. 


\section{La década de 1990: regulaciones neoliberales}

Durante los 1990, el contexto político y económico era altamente favorable para la difusión de la biotecnología en Brasil. Mientras los agricultores se encontraban en una situación financiera precaria debido a la reducción de subsidios gubernamentales, el gobierno necesitaba mejorar su balanza comercial. La tecnología genéticamente modificada prometía más ganancias y competitividad, con lo que se alinearían los intereses de agricultores y gobierno (Pelaez y Schmidt, 2000). Esto abrió camino para cambios legales que incorporasen las reglas del globalismo neoliberal indispensables a la introducción de las semillas transgénicas.

Luego de firmar los acuerdos de libre comercio de la Organización Mundial del Comercio (OMC) en 1994, Brasil aprobó una Ley de Bioseguridad en 1995 y en 1996 el parlamento incorporó el Acuerdo sobre los Aspectos de los Derechos de Propiedad Intelectual relacionados con el Comercio (TRIPS) en la Ley de Propiedad Industrial. En 1997 sus implicaciones en el área de semillas fueron garantizadas legalmente en la Ley de Protección de Variedades Vegetales y un proyecto para una nueva ley de semillas fue lanzado. Esta ley obstaculizó el desarrollo del fitomejoramiento en la dirección descrita por Kloppenburg (2004); es decir, inclinó el balance hacia el sector privado y la mercantilización de semillas, al aumentar las posibilidades para la protección de los DPI y la formalización y concentración del mercado de semillas.

La oposición parlamentaria y los movimientos sociales debatieron estos acontecimientos y lograron ciertas concesiones. Durante la votación de las nuevas leyes en DPI y variedades vegetales, los puntos clave de disputa abarcaron la apropiación de semillas. Diversos movimientos agroecológicos y agrónomos influenciaron la opción brasileña por adoptar la Convención UPOV 78 en vez de la UPOV 91 (defendida por las corporaciones de semillas). Esta elección garantizó los derechos del agricultor 
para reutilizar semillas y la ley brasileña estableció una protección especial para pequeños agricultores.

\section{Cultivos transgénicos ilegales en el sur de Brasil: una zona de disputas}

En Brasil, la primera gran coalición contra los cultivos transgénicos fue subnacional y se suscitó en Rio Grande do Sul, en la frontera con Argentina, a consecuencia del contrabando de semillas. El gobernador recién electo, preocupado por la agricultura familiar y los movimientos campesinos, declaró su intención de hacer Rio Grande do Sul una zona libre de transgénicos, al aplicar una legislación más estricta que la nacional (Pelaez y Schmidt, 2000). El 20 de agosto de 1999, movimientos sociales y ONG locales lanzaron el primer manifiesto público de la sociedad civil en Brasil; demandaron que el Estado, en todos sus niveles (federal, estatal y local), suspendiera la producción o importación de OGM, con base en el principio de precaución.

Ese mismo año, el Movimiento de los Trabajadores Rurales sin Tierra (MST) anunció un plan de ocupar las haciendas que plantasen semillas transgénicas en Rio Grande do Sul durante los próximos dos años (Samora, 1999). Aunque tiempo después el plan fue nacionalizado (Silva, 2000), el estado de Rio Grande do Sul se mantuvo en zona de disputas. En 2001, Porto Alegre fue sede de la primera edición del Foro Social Mundial, en ella participaron mil activistas del MST, quienes arrancaron plantas de soja transgénica de campos experimentales de Monsanto (Traumann, 2001).

En el estado vecino, Paraná, la oposición a los transgénicos también tomó impulso. En 2003, el gobernador electo prohibió la importación y exportación de semillas transgénicas a través del puerto estatal y mediante una ley declaró a Paraná zona libre de transgénicos. Los estados de Mato 
Grosso y Mato Grosso do Sul, que experimentaban un fuerte crecimiento en la producción soyera, respondieron a esas acciones en un juicio legal (Tortato, 2003). La Corte Suprema juzgó ese hecho como inconstitucional, argumentó que el gobierno federal poseía la autoridad para decidir en materia de OGM. En adición, los medios de comunicación habían convertido la política nacional sobre OGM en objeto de fuertes disputas.

\section{Organizaciones nacionales, movilización legal y una campaña nacional contra los transgénicos}

La movilización legal fue una estrategia crucial en las luchas contra los cultivos transgénicos en Brasil, además el marco jurídico permitió una serie de provisiones con las que la sociedad legal pudiese argumentar que la aprobación de semillas transgénicas amenazaba los derechos. La demanda por el cumplimiento de las leyes existentes o por el establecimiento de un marco legal apropiado convergió con el espíritu de la época, a saber, de la consolidación democrática, en el cual la ley y su ejercicio se convirtieron en objetos de pugnas. Legalidad y democracia eran una demanda recurrente de los movimientos sociales en la campaña brasileña, al igual que los derechos del consumidor y la protección ambiental. La sociedad civil tuvo éxito en transformar el tema en un problema político, sobre todo por la existencia de movimientos sociales organizados a nivel nacional, los cuales desarrollaban actividades de base.

Greenpeace fue la primera en organizar una acción directa contra los cultivos transgénicos en Brasil. En 1997, sus miembros bloquearon un navío con una carga de soja transgénica. A causa de la difusión que se le dio en las noticias internacionales, el acto atrajo de inmediato la atención de la media nacional (entrevista con Greenpeace Brasil, 2012). Esta organización también promovió una acción civil pública y demandó respeto con el Código del Consumidor (entrevista con exmiembro de IDEC, 2013). 
El 24 de septiembre de 1998, cuando la Comisión Técnica Nacional en Bioseguridad (CTNBio) emitió una opinión técnica favorable para la aprobación de la soja transgénica, el Instituto Brasileño de Defensa del Consumidor (IDEC) estableció una orden judicial para suspender la aprobación inminente: «Nosotros demandamos una orden para prevenir la liberación hasta que fuesen creadas normas para evaluar los riesgos a la salud y el medio ambiente y una regla de etiquetaje para garantizar los derechos del consumidor» (entrevista con exmiembro de IDEC, 2013). El poder judicial aprobó la orden ese mismo día y funcionó como una moratoria por los próximos cinco años. La acción judicial puso en peligro los planes de la industria biotecnológica en Brasil, en su coalición con sectores gubernamentales y científicos (entrevista con exmiembro de IDEC, 2013). Significó «un gran boom, una gran señal de alerta» (entrevista con ASPTA, 2012) que motivó a otras organizaciones a unir sus esfuerzos y lanzar la campaña nacional Por un Brasil Libre de Transgénicos, en 1999. Los movimientos afiliados a la Vía Campesina, incluyendo el MST, se unirían más tarde en una campaña que unificó movimientos urbanos y agrarios.

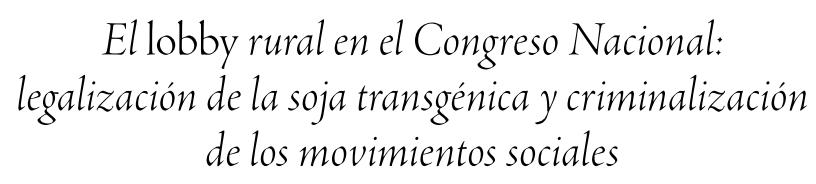

La batalla judicial tuvo grandes consecuencias para el gobierno, al instaurar desavenencias internas que se mantendrían los años siguientes. El Ministerio de Agricultura no aceptó la moratoria, pues había estado negociando con Monsanto la introducción del producto en el país hacía tres años, e incitó a la empresa multinacional a registrar las semillas como una variedad vegetal, a pesar de la imposibilidad de hacer la soja RR legalmente disponible para la siembra de 1999/2000 (Leite, 1998). Paralelamente, la Empresa Brasileña de Investigación Agropecuaria (Embrapa), 
subordinada al Ministerio de Agricultura, multiplicó semillas de soja transgénica, siguiendo un acuerdo de cooperación con Monsanto (Rippardo y Murakawa, 2000). Agricultores del Sur de Brasil que vivían en la frontera empezaron a contrabandear semillas transgénicas de Argentina para Brasil. En 1998, la policía confiscó soja transgénica plantada de forma ilegal. ${ }^{3}$

En 2003, el gobierno federal emitió una medida provisoria en la que aprobaba la comercialización nacional de los cultivos ilegales. Insatisfecho, el lobby agrario demandó una solución permanente para el problema, y el gobierno envió un proyecto de una nueva Ley de Bioseguridad al parlamento, un bastión de la bancada rural, mismo que se convirtió en la principal tribuna para las disputas acerca de los cultivos transgénicos durante los próximos dos años. El MST protestó y a lo largo de cuarenta días acampó frente al congreso. La campaña Por un Brasil Libre de Transgénicos, por medio de su alianza con la Coalición Parlamentaria por la Bioseguridad y el Principio Precautorio, incluyó algunos artículos de transparencia y participación pública en las decisiones respecto a los transgénicos, así como un cierto margen para una interpretación precautoria. El resultado final fue un texto que incorporó casi todas las demandas de la coalición pro OGM.

Lanzada en 2005, la Ley de Bioseguridad aclaró el estado legal de la soja GM y puso fin a los efectos de la moratoria de 1998. Aunque ya había plantíos ilegales, la legalización de la soja transgénica fue un paso decisivo para su fuerte incremento y dominancia en los campos brasileños. Eso también silenció las controversias en los niveles subnacionales, reafirmó que la competencia de decidir acerca del tema recaía en el nivel nacional, con consecuencias que afectaban a todos los miembros de la federación.

${ }^{3}$ Para la cosecha de 2002-2003, «estimaciones de varias fuentes del mercado evalúan que alrededor de 70 por ciento de la producción en RS, estimada en 8.4 millones de toneladas, fue sembrada con soja transgénica» (Embrapa Soja, 2003: 4). 
De modo simultáneo al debate sobre la soja transgénica, el lobby rural tomó medidas para criminalizar los movimientos sociales del campo que peleaban por la reforma agraria y por un modelo alternativo de desarrollo agrario. Los congresistas establecieron dos Comisiones de Investigación Parlamentaria con el propósito de indagar las actividades del MST -CMPI da Terra, en 2003 (Lupion, 2005), y CPI das ONG, en 2006-, y la transferencia de recursos públicos a las ONG, a fin de atacar la alianza entre el gobierno y los movimientos sociales. Ello influyó en la agenda mediática, pues la intención del MST era diseminar una imagen negativa del movimiento, de ahí que fueran acusados de robar y desviar dinero público para practicar actos de «vandalismo» e «invadir» tierras y propiedad privada. Inmersos en esas dificultades y teniendo que retroceder en su principal lucha, la reforma agraria, el MST disminuyó sus acciones contra los transgénicos.

\section{Desafiando la comisión de expertos: democracia}

Una vez que el congreso delegó el poder de decisión a la comisión de expertos, ésta se convirtió en el próximo blanco de la campaña Por un Brasil Libre de Transgénicos. De igual forma, se desafió el cierre tecnocrático del debate al construir argumentos basados en «la democracia», «el interés público», «la participación», «la transparencia»y «la responsabilidad pública». Las prácticas fueron calificadas como «puertas cerradas», «ilegales» e ilegítimas (entrevista con AS-PTA, 2012).

En 2007, tres variedades de maíz transgénico fueron aprobadas para uso comercial. El Movimiento de Mujeres Campesinas (MMC) interrumpió la reunión de expertos con mujeres embarazadas que cargaban carteles con la consigna «Mi hijo no es un conejito de indias». Distintos activistas formularon una petición al presidente y entablaron un juicio para suspender la decisión, con ello esperaban ganar alguna influencia 
sobre el proceso político nacional. Sin embargo, el maíz transgénico fue aprobado de modo definitivo en 2008. Eso marcó un salto considerable en la aprobación de otras semillas transgénicas y también la conversión de Brasil en un gran productor mundial de granos genéticamente modificados. ${ }^{4}$

A medida que la política nacional de OGM se tornó impermeable para la influencia de los movimientos sociales, la campaña Por un Brasil Libre de Transgénicos hizo asimismo intervenciones y protestas en la arena internacional, afirmó que la autorización de cultivos transgénicos en Brasil no cumplía con sus obligaciones internacionales bajo el Protocolo de Cartagena. Este intento de alcanzar un «efecto búmeran» (Keck \& Sikkink, 1998) a través de alguna sanción por parte del Convenio sobre Diversidad Biológica no encontró apoyo en el nivel internacional.

\section{Conclusiones}

Borras (2009), en su llamado a los estudios críticos en torno a las transformaciones agrarias, advierte acerca del peligro de despolitizar el cambio agrario, ofuscando las luchas políticas involucradas. No es el caso de las disputas relacionadas con los cultivos transgénicos en Brasil. Por más de una década, los movimientos sociales como Por un Brasil Libre de Transgénicos sostuvieron una campaña que siguió de cerca y aspiró a influenciar el proceso político nacional. Se dedicaron a contactar ministerios, entraron en batallas legislativas, en la comisión de expertos e

${ }^{4}$ Hasta 2007 se habían aprobado una variedad de soja, una de algodón y tres de maíz. Desde 2008 ha habido un fuerte incremento en estos números. Hoy existen seis variedades de soja aprobadas, 29 de maíz, 12 de algodón, una de frijol, una de eucaliptos (CTNBio 2015). En 2009, Brasil superó a Argentina y se convirtió en el segundo productor mundial de cultivos transgénicos. 
hicieron demandas directamente al presidente. Muchas veces, ellos atribuían a sus demandas sentidos relativos a la legalidad y la democracia, solicitaban un debido proceso democrático en las decisiones relativas a la política de OGM. Si bien el principal objetivo había sido el gobierno nacional, también participaron con acciones en las escalas subnacional e internacional.

En el otro lado de la disputa, una red de actores formada por elites agrarias, corporaciones multinacionales semilleras y químicas, científicos, políticos alineados al lobby rural y algunos formuladores de políticas se involucraron activamente en la construcción de un contexto político e institucional favorable a la transformación de Brasil en un productor global de cultivos transgénicos. Entre las estrategias adoptadas, agricultores, agencias estatales y empresas semilleras actuaron de manera ilegal, pues reprodujeron y comercializaron soja transgénica no aprobada, y así crearon una situación de faits accomplis (hecho consumado). La soja transgénica ilegal se convirtió en el cultivo principal en los campos de los estados brasileños en la frontera con Argentina y eso indujo esfuerzos para legalizar una situación de facto. Para eso, la coalición pro OGM trabajó para cambiar la ley así como las reglas de funcionamiento de la comisión de expertos responsable por la aprobación de OGM. Ello revela la importancia de la política y del Estado nacional en la regulación de la producción y el comercio de cultivos transgénicos en línea con los intereses del agronegocio nacional y global.

Finalmente, a pesar de la dimensión global e internacional de la cuestión, la batalla alrededor de la adopción de la agrobiotecnología en Brasil fue librada a nivel del Estado nacional, el cual movilizó tanto actores en favor de los transgénicos como movimientos sociales y ONG en contra. El Estado nacional tuvo un papel central al mediar estas disputas en las arenas ejecutiva, judicial y legislativa. El marco legal nacional y su interpretación fueron cruciales: por un lado se exigió su aplicación y por el 
otro se trabajó en cambiarlo con la finalidad de que sus intereses no fuesen cuestionados. El caso brasileño es un ejemplo valioso para analizar el globalismo neoliberal en los procesos (disputados) de su traducción y operacionalización en contextos nacionales. En Brasil y otros lugares, la adopción y la aplicación de leyes y regulaciones ha beneficiado actores corporativos y elites agrarias para la institucionalización de un régimen alimentario basado en la biotecnología.

\section{Referencias}

Anderson, P. (2004). «What Rights Are Eclipsed When Risk Is Defined by Corporatism?: Governance and GM Food». Theory, Culture E Society 21(6), pp. 155-170.

Borras, S.M. (2009). «Agrarian Change and Peasant Studies: Changes, Continuities and Challenges. An Introduction». Journal of Peasant Studies 36(1), pp. 5-31.

Borras Jr, S.M., Edelman, M. \& Kay, C. (2008). Transnational Agrarian Movements Confronting Globalization. Chichester: John Wiley \& Sons.

Cáceres, D.M. (2015). «Accumulation by Dispossession and Socio-Environmental Conflicts Caused by the Expansion of Agribusiness in Argentina». Journal of Agrarian Change 15(1), pp. 116-147.

CTNBio (2015). Resumo Geral de Plantas Geneticamente Modificadas Aprovadas Para Comercialização, http://www.ctnbio.gov.br/upd_blob/0002/2086.pdf Embrapa Soja (2003). Cronologia Do Embargo Judicial, http://www.cnpso.embrapa.br/download/cronologia_sojarr.pdf

Fitting, E.M. (2011). The Struggle for Maize: Campesinos, Workers, and Transgenic Corn in the Mexican Countryside. Durham: Duke University Press. 
Friedmann, H. \& McMichael, P. (1989). «Agriculture and the State System: The Rise and Decline of National Agricultures, 1870 to the Present». Sociologia Ruralis 29(2), pp. 93-117.

Fuglie, K., Heisey, P., King, J. \& Schimmelpfennig, D. (diciembre, 2012). «Rising Concentration in Agricultural Input Industries Influences New Technologies». Amber Waves, USDA Economic Research Service.

Glover, D. (2010). «The Corporate Shaping of GM Crops as a Technology for the Poor». Journal of Peasant Studies 37(1), pp. 67-90.

Góngora Mera, M. y Motta, R. (2014). «El Derecho Internacional y la Mercantilización Biohegemónica de la Naturaleza: la Diseminación Normativa de la Propiedad Intelectual sobre Semillas en Colombia y Argentina». En Göbel, B., Góngora Mera, M. y Ulloa, A. (eds.), Desigualdades socioambientales en América Latina. Bogotá: Universidad Nacional de Colómbia, pp. 395-433.

Gras, C. y Hernández, V. (2008). «Modelo productivo y actores sociales en el agro argentino». Revista Mexicana de Sociología 70(2), pp. 227-259.

James, C. (2015). Global Status of Commercialized Biotech/GM Crops: 2014. 49. Ithaca, NY: ISAAA.

Jasanoff, S. (2005). Designs on Nature: Science and Democracy in Europe and the United States. Princeton: Princeton University Press.

Keck, M.E. \& Sikkink, K. (1998). Activists beyond Borders: Advocacy Networks in International Politics. Cambrigde: Cambridge University Press.

Kinchy, A.J. (2012). Seeds, Science, and Struggle: The Global Politics of Transgenic Crops. Cambridge: MIT Press.

Kloppenburg, J.R. (2004). First the Seed: The Political Economy of Plant Biotechnology, 1492-2000. Madison: University of Wisconsin Press.

Lapegna, P. (2014). «Global Ethnography and Genetically Modified Crops in Argentina: On Adoptions, Resistances, and Adaptations». Journal of Contemporary Ethnography 43(2), pp. 202-227. 
Leite, M. (octubre, 1998). «Para Empresa, Planta é Idêntica». Folha de São Paulo 4. Lupion, A. (2005). Relatório Dos Trabalhos da CPMI da Terra, http://www. senado.gov.br/comissoes/CPI/RefAgraria/CPMITerra.pdf

McMichael, P. (2009). «A Food Regime Genealogy».Journal of Peasant Studies 36(1), pp. 139-169.

Motta, R. (2016). Social Mobilization, Global Capitalism and Struggles over Food: A Comparative Study of Social Movements. London, New York: Routledge. Motta, R. (2015). «Transnational Discursive Opportunities and Social Movement Risk Frames Opposing GMOs». Social Movement Studies 14(5), pp. 576-595.

Newell, P. (2006). «Corporate Power and «Bounded Autonomy in the Global Politics of Biotechnolgoy». In Falkner, R. (ed.), The International Politics of Genetically Modified Food: Diplomacy, Trade, and Law. Basingstoke: Palgrave Macmillan, pp. 67-85.

Otero, G. (2004). «Global Economy, Local Politics: Indigenous Struggles, Civil Society and Democracy». Canadian Journal of Political Science/Revue Canadienne de Science Politique 37(2), pp. 325-346.

Pechlaner, G. \& Otero, G. (2008). «The Third Food Regime: Neoliberal Globalism and Agricultural Biotechnology in North America». Sociologia Ruralis 48(4), pp. 351-71.

Pelaez, V. y Schmidt, W. (2000). «A difusão dos OGM no Brasil: imposição e resistências». Estudos Sociedade e Agricultura 14, pp. 5-31.

Pengue, W. (2005). «Transgenic Crops in Argentina: The Ecological and Social Debt». Bulletin of Science, Technology E Society 25(4), pp. 314-322.

Rippardo, S. y Murakawa, F.E. (julio 18, 2000). «Pesquisa Da Embrapa Favorece Monsanto». Folha de São Paulo.

Samora, R. (diciembre 19, 1999). «MST Anuncia Invasão de Fazendas com Transgênicos». Folha de São Paulo. 
Schurman, R. \& Munro, W. (2010). Fighting for the Future of Food: Activists versus Agribusiness in the Struggle over Biotechnology. Minneapolis: University of Minnesota Press.

Silva, E. (agosto 12, 2000). «Reuniao do MST Prega Invasão e Queimada». Folba de São Paulo.

Tarrow, S. (2011). Power in Movement: Social Movements and Contentious Politics. Cambridge: Cambridge University Press.

Tortato, M. (octubre 28, 2003). «Requiäo Fecha Paranaguá para Transgênico». Folha de São Paulo, http://www1.folha.uol.com.br/fsp/dinheiro/fi2810 200324.htm

Traumann, T. (enero 27, 2001). «MST Queima Soja Transgênica da Monsanto». Folha de São Paulo. 